\title{
Why Chronic Pancreatitis Inpatients Should Not Be Impatient
}

\author{
John G. Lieb ${ }^{1}$. Chris E. Forsmark ${ }^{1}$ \\ Published online: 19 June 2020 \\ (c) Springer Science+Business Media, LLC, part of Springer Nature 2020
}

Healthcare outcomes may be adversely affected by numerous factors beyond the disease itself, including inadequate access to care, disparities in health care delivery, cost, lack of family and social support, coexistent substance abuse or mental illness, poor compliance, and many others. In this issue of Digestive Diseases and Sciences, Akanbi et al. [1] studied the frequency and predictors of patients with chronic pancreatitis leaving the hospital against medical advice (AMA). Of course, a small percentage of all patients admitted to the hospital for any reason are at risk of leaving the hospital AMA. Although there are several reasons why patients leave the hospital AMA, this early departure from medical care is often associated with an attendant increase in morbidity [2] and mortality [3], as well as an increase in readmission rates to the hospital $[4,5]$. The investigators estimated the prevalence and predictors of leaving AMA among hospitalized chronic pancreatitis patients in the USA, hypothesizing that alcohol, substance abuse and mental health disorders might be more common in these patients and could be risk factors for leaving AMA. In order to test their hypothesis, the investigators retrospectively examined deidentified information on hospital discharges from 2008 to 2014 from the National Inpatient Database (NIS), which tracks 7 million discharges per year and is a representative sampling of the US hospitalized population.

The investigators analyzed the racial, demographic, geographic, and socioeconomic characteristics, length of hospital stay, total adjusted charges, type of insurance, day of the week of the admission, medical comorbidities and preexisting conditions present in these patients. Comorbid conditions were analyzed using ICD9 codes, including alcohol use, tobacco use, other substance use, depression, psychosis,

John G. Lieb

john.lieb@medicine.ufl.edu

Chris E. Forsmark

chris.forsmark@medicine.ufl.edu

1 Division of Gastroenterology, Hepatology, and Nutrition, University of Florida, Box 100214, 1329 SW 16th St, Suite 5251, Gainesville, FL 32610-0214, USA
HIV status, biliary stones, acute pancreatitis, and undergoing endoscopic or surgical biliary and pancreatic procedures. Moreover, characteristics of the hospitals where the patients were admitted were tracked by region, size, location (urban or rural), and by mission (academic vs nonacademic, for profit vs. not for profit). The initial reason for hospitalization was not analyzed, although management of flares of chronic abdominal pain is the reason for admission in the vast majority of patients with chronic pancreatitis [6].

The primary outcome of interest was leaving the hospital AMA. Readmission rates and causes for those readmissions, which would have been interesting, were not tracked. A post hoc analysis adjusting for smoking/alcohol abuse and etiology of the chronic pancreatitis was performed. Patients admitted with gastroparesis were chosen as an additional comparison group. The database revealed that 87,068 patients were discharged with the diagnosis of chronic pancreatitis between 2012 and 2014, with $3.45 \%$ of those discharges being AMA. Univariate analysis revealed that patients who left AMA were more likely to be younger, male, African-American or Latino, in the lowest quartile of annual income, receiving Medicaid or uninsured, admitted from the emergency ward to a private for-profit hospital, and from the southern and northeastern USA. Patients with chronic pancreatitis who left the hospital AMA were also more likely to smoke, use alcohol and illicit drugs and were less likely to have undergone endoscopic or surgical procedures or have preexisting medical or psychiatric comorbidities (other than HIV and "psychosis"), than those who did not leave AMA. Understandably, when observed from the narrow perspective of a single hospitalization, each hospital stay was less costly and shorter among those who left AMA. According to multivariable analysis, most of these variables remained predictors of AMA discharge with the exception of race, smoking status, and the presence of concomitant acute pancreatitis likely due to biliary stones. When compared with all patients hospitalized in the USA from 2008 to 2012, the rate of AMA discharge for chronic pancreatitis patients was more common and increased at a greater rate 
than for other patients (from $3 \%$ to $3.7 \%$ vs from $1.1 \%$ to $1.3 \%$ for all other patients).

According to post hoc analyses, those patients using alcohol and/or tobacco were more likely to leave AMA (4.6\% vs. $2.0 \%$ ) than patients who did not use alcohol and tobacco. Idiopathic chronic pancreatitis patients were the least likely to leave AMA (1.37\%). Chronic pancreatitis patients were more likely to leave AMA than were gastroparesis patients (3.45\% vs. $2.33 \%)$. Interestingly, many of the factors that predicted leaving the hospital AMA were similar for both chronic pancreatitis and gastroparesis.

Patients with chronic pancreatitis appear to leave the hospital AMA more commonly than do patients with many other medical conditions a rate that appears to be increasing over time. The authors identify potential predictors of this adverse health outcome, but they do not identify strategies to reduce or prevent patients from making the decision to leave the hospital AMA. Many of the predictors seem obvious, such as rather high rates of associated alcohol use disorder, lack of insurance, or poverty. There may be a multitude of other confounding explanations for leaving AMA; two in particular, deserve mention. In our experience, increasing frustration with the lack of effective treatment options for chronic pancreatitis pain drives many patients to give up on the medical system. Perhaps even more importantly, healthcare workers exhibit a lack of understanding regarding the pathophysiology of chronic pancreatitis. For instance, many clinicians are not aware that in patients with end-stage chronic pancreatitis, amylase and lipase elevations are absent, despite pain flares. Furthermore, cross-sectional imaging will often not reveal acute inflammation in the presence of chronic changes. Finally, many clinicians have the common misperception that all chronic pancreatitis is due to alcohol [7]. In this situation, patients are routinely told that they cannot be experiencing a painful flare, and are accused of pain medication-seeking behavior. They are also often accused repeatedly of being alcoholics. Therefore, most patients with longstanding painful chronic pancreatitis avoid the hospital and emergency room at all costs, only coming in when symptoms are overwhelming. They might also be more likely to leave at the first sign of improvement. A unified, single-message hospitalist/nursing/GI/surgery team is most effective for better communicating the intentions and goals of the team to chronic pancreatitis patients-not just regarding opiate use for pain, but for all aspects of care. Further, early on in the admission we need to ask patients directly, "what might motivate you to leave the hospital before being medically cleared?" or "what can we do to ensure you stay hospitalized until we recommend discharge?"

Going forward, therapeutic options for those suffering with chronic pancreatitis need to be improved [8]. Efficacious therapies that are better tolerated would reduce the number of disgruntled patients who choose to leave AMA. In the meantime, we can identify those chronic pancreatitis patients at high risk of leaving AMA early in their hospitalizations and make a concerted effort to tailor existing therapies for their specific needs, as well as provide supportive counseling resources to help them better manage their disease.

\section{References}

1. Akanbi O, Adejumo AC, Soliman M, et al. Chronic pancreatitis patients who leave against medical advice: prevalence, trend and predictors. Dig Dis Sci. (Epub ahead of print). https://doi. org/10.1007/s10620-020-06279-2.

2. Bhoomadevi A, Baby TM, Keshika CC. Factors influencing discharge against medical advice (DAMA) cases at a multispecialty hospital. J Family Med Prim Care. 2019;8:3861-3864.

3. Glasgow JM, Vaughn-Sarrazin M, Kaboli PJ. Leaving Against Medical Advice (AMA): risk of 30-day mortality and hospital readmission. J Gen Intern Med. 2010;25:926-929.

4. Southern WN, Nahvi S, Arnsten JH. Increased risk of mortality and readmission among patients discharged against medical advice. Am J Med. 2012;125:594-602.

5. Saab D, Nisenbaum R, Dhalla I, et al. Hospital readmissions in a community-based sample of homeless adults: a Matched-cohort study. J Gen Intern Med. 2016;31:1011-1018.

6. Mullady DK, Yadav D, Amann ST, et al. Type of pain, painassociated complications, quality of life, disability, and resource utilization in chronic pancreatitis: a prospective cohort study. Gut. 2011;60:77-84.

7. Kleff J, Whitcomb DC, Shimosegawa T, et al. Chronic pancreatitis. Nat Rev Dis Primers. 2017;3:17060.

8. Forsmark CE, Andersen DK, Farrar JT, et al. Accelerating the drug delivery pipeline for acute and chronic pancreatitis: summary of the working group on drug development and trials in chronic pancreatitis at the national institute of diabetes and digestive and kidney diseases workshop. Pancreas. 2018;47:1200-1207.

Publisher's Note Springer Nature remains neutral with regard to jurisdictional claims in published maps and institutional affiliations. 